\title{
Cortisol levels and sleep patterns in infants with orofacial clefts undergoing surgery
}

This article was published in the following Dove Press journal:

Neuropsychiatric Disease and Treatment

15 October 2014

Number of times this article has been viewed

\author{
Andreas A Mueller ${ }^{1,2}$ \\ Nadeem Kalak ${ }^{3}$ \\ Katja Schwenzer- \\ Zimmerer ${ }^{1,2}$ \\ Edith Holsboer-Trachsler ${ }^{3}$ \\ Serge Brand ${ }^{3,4}$ \\ 'Craniomaxillofacial Surgery, \\ University of Basel and University \\ Hospital of Basel, Basel, Switzerland; \\ ${ }^{2}$ Hightech Research Center of \\ Craniomaxillofacial Surgery, \\ University of Basel, Basel, Switzerland; \\ ${ }^{3}$ Psychiatric Clinics of the University \\ of Basel, Center for Affective, \\ Stress, and Sleep Disorders, Basel, \\ Switzerland; ${ }^{4}$ Department of Sport \\ and Health Science, Division of Sport \\ Science, University of Basel, Basel, \\ Switzerland
}

\begin{abstract}
Background: Traumatic events during early infancy might damage infants' psychobiological functioning, such as sleep and cortisol secretion. Infants born with orofacial clefts (OFCs) undergo functional, anatomical, and aesthetic surgery. The aim of the present study was to determine whether infants with OFC and undergoing OFC surgery show deteriorated sleep and cortisol secretion compared with healthy controls and with their presurgery status.
\end{abstract}

Methods: A total of 27 infants with OFC (mean age: 22 weeks) and 30 healthy controls (mean age: 23 weeks) took part in the study. For infants with OFC, sleep actigraphy was performed and saliva cortisol was analyzed 5 days before, during, and 5 days after surgery. For controls, sleep and saliva cortisol were assessed similarly, except for the period taken up with surgery.

Results: Compared with healthy controls, infants with OFC undergoing OFC surgery did not differ in sleep and cortisol secretion. Their sleep and cortisol secretion did deteriorate during the perisurgical period but recovered 5 days postsurgery.

Conclusion: In infants with OFC undergoing corrective surgery, the pattern of results for sleep and cortisol suggests that OFC surgery does not seem to constitute a traumatic event with long-term consequences.

Keywords: cortisol, sleep, orofacial cleft, surgery, infants

\section{Introduction}

Orofacial clefts (OFCs) are the most frequent birth defects worldwide. One in 500-700 newborns suffers from an OFC. ${ }^{1-3}$ Prevalence rates vary as a function of ethnic, geographical, and socioeconomic factors and mothers' general health status. The term "orofacial cleft" covers a wide range of disorders affecting the lips and oral cavity. Typically, cleft lip (CL) (World Health Organization International Classification of Diseases, Tenth Edition [ICD-10]: Q36.-), CL and cleft palate (CP) (ICD-10: Q37.-), and CP (ICD-10: Q35.-) are distinguished. Additionally, OFCs may be left- or rightsided (unilateral) or both-sided (bilateral). OFCs result from a failure during embryonic development. Specifically, between the fourth and 17 th weeks of embryonic development, the closure mechanisms for the facial and oral cavity fail. At the very beginning of embryonic development, the embryo is particularly sensitive to adverse substances, while women are not always aware of being pregnant at this early stage of pregnancy. ${ }^{1,4}$ Overall, there is agreement that factors related to genetic, environment, and gene-environment interaction explain best the occurrence of OFCs. ${ }^{1,3}$

Numerous studies confirm the importance of sleep and cortisol secretion in regulating physiological and psychological homeostasis, and this also holds true for infants. However, infants' sleep schedules and cortisol secretion evolve over the first 12 months of life.
Psychiatric Clinics of the University of Basel, Center for Affective, Stress and Sleep Disorders, Wilhelm Klein-Strasse 27, 4027 Basel, Switzerland

Tel $+416 \mid 3255114$

Fax $+4|6| 3255553$

Email serge.brand@upkbs.ch
Dovepress

http://dx.doi.org/10.2147/NDT.S71785
Neuropsychiatric Disease and Treatment 2014:10 1965-1972

(c) (i) (c) 2014 Mueller et al. This work is published by Dove Medical Press Limited, and licensed under Creative Commons Attribution - Non Commercial (unported, v3.0)

1965 permission from Dove Medical Press Limited, provided the work is properly attributed. Permissions beyond the scope of the License are administered by Dove Medical Press Limited. Information on how to request permission 
With regard to a regular sleep/wake pattern, circadian and ultradian processes begin to develop in the newborn period, and a circadian rhythm emerges at around 2-3 months of age, ${ }^{5}$ with a synchronization of the sleep/wake cycle in relation to light/dark cycles and social stimuli such as feeding. 5,6 The period from 8 to 16 weeks of life is also marked by major behavioral reorganization in infants. ${ }^{7}$ Circadian rhythmicity, sleep, and endocrine processes are associated, and more robust patterns are seen at approximately 3 months of age. ${ }^{7}$

Within the neuronal and neuroendocrine system, the activity of the hypothalamic-pituitary-adrenocortical (HPA) system demands particular attention. The most prominent outcome measure of HPA axis activity is cortisol secretion. ${ }^{8,9}$ The HPA axis enables the human organism to cope specifically and individually with internal and external stressors. ${ }^{9,10}$ In this regard, Gruber et $\mathrm{al}^{11}$ demonstrated a significant neuroendocrine stress response in infants with well-compensated congenital cardiac disease undergoing cardiac surgery but without adverse postoperative outcomes. In a similar vein, Nakamura et al ${ }^{12}$ compared HPA axis reactivity to surgical stress in 19 neonates, 19 infants, and 20 preschool children undergoing major thoracic and abdominal surgery. Regardless of age, cortisol levels reached a peak just after surgery. These results suggest that in infants aged $<6$ months, the HPA axis predominantly reacts in response to physical rather than psychological stressors. ${ }^{9,10,13}$

Additionally, cortisol peak values seem significantly lower in neonates than in infants and preschool children ${ }^{8}$ (see also Mantagos et $\mathrm{al}^{14}$ for opposite results). For this reason, it has been proposed that the neuroendocrine transmission system in newborns is still immature and that cortisol secretion periodicity is established postnatally. Indeed, in the first 4 postnatal weeks, cortisol secretion periodicity increases from 6.4 hours with two daily peaks to 11.2 hours with one daily peak. ${ }^{9}$ By about 8 weeks of age, the HPA system appears to be sufficiently mature to produce a 24 -hour periodicity, but this periodicity is not clearly synchronized with the light/ dark cycle. Synchronization and coordination with emerging circadian rhythmicity in nighttime sleep patterns increase from 8 weeks onwards, and adult-like coordination of HPA periodicity, light/dark cycle, and nighttime sleep rhythms begins to emerge by $12-16$ weeks. With this synchronization, basal cortisol levels increasingly become the peak levels seen during the day. ${ }^{15}$ Therefore, the conjunction of increased circadian and day/night organization at around 3 months and the change in cortisol secretion to a 24-hour periodicity suggest that these two phenomena are functionally related. In this respect, Larson et al ${ }^{16}$ showed that infants who were reported to sleep at least 6 hours or more continuously through the night had a more marked circadian variation in cortisol production. Accordingly, infants with an early morning peak cortisol level were significantly older, and they were also more likely to sleep for 6 hours or more without signaling during the night.

Overall, we note that a close association between sleep pattern and HPA axis activity has repeatedly been shown in adults, ${ }^{17,18}$ preschool children, ${ }^{19}$ and infants, ${ }^{20,21}$ with poor sleep related to increased cortisol secretion. However, nothing is known about the relationship between sleep and the HPA axis activity in infants with OFC and undergoing OFC surgery. The aim of the present study was therefore to fill this gap in our knowledge and to compare data from such infants with data from healthy controls. We believe that these issues are of particular concern because, generally, parents of infants with OFC are concerned about their infants' long-term development, including the extent to which OFC surgery might negatively affect their psychophysiological development. This holds particularly true because findings from animal and preclinical studies highlight the potential relevance of early stress and cortisol secretion for later onset of psychiatric disorders. ${ }^{22}$

The following three hypotheses were formulated. First, following Gruber et $\mathrm{al}^{11}$ and Nakamura et $\mathrm{al}^{12}$ and others, ${ }^{9,10,13}$ we expected increased cortisol secretions in infants with OFC immediately during peri- and postsurgery, but not 5 days before or 5 days after surgery. Accordingly, we expected that cortisol secretion in infants with OFC 5 days before surgery would not differ from the level 5 days postsurgery or from the cortisol secretion of healthy controls. Third, following Brand et $\mathrm{al}^{21}$ we expected associations between poor sleep as objectively assessed via actigraphy and increased cortisol secretion.

\section{Methods}

\section{Sample}

A total of 27 infants (13 female infants [48\%]; mean age at assessment and surgical intervention: 22 weeks; standard deviation $[\mathrm{SD}]=1.5$ weeks) with $\mathrm{OFC}$ and undergoing OFC surgery in the Craniomaxillofacial Surgery Center, University Hospital Basel (Basel, Switzerland), took part in the present study. All children were full term, mean birth weight was 3,510 g ( $\mathrm{SD}=390.20 \mathrm{~g})$, and mean Apgar scores were $7-10 / 8-10 / 8-10$. Four of the 27 children (14.8\%) were born by cesarean section. The following OFCs were observed: $16 \mathrm{CL}$ and CP (ICD-10: Q37; 13 unilateral and three bilateral) three CP (ICD-10: Q35), and eight CL (unilateral; ICD-10: Q36). 
In parallel, a total of 30 healthy infants (15 females [50\%]; mean age at assessment: 24 weeks; $\mathrm{SD}=2.1$ weeks) were assessed. Children were full term, mean birth weight was $3,800 \mathrm{~g}(\mathrm{SD}=400 \mathrm{~g})$, and mean Apgar scores were 9-10/9-10/9-10. Five of the 30 children (16.7\%) were born by cesarean section. These healthy children were recruited via word of mouth among staff from the university hospitals and personal connections.

All infants' parents were fully informed about the aims and the procedure of the study and gave written informed consent. The study was conducted in accordance with the Declaration of Helsinki, and the experimental protocol was approved by the local ethics committee of Basel (Basel, EKBB: 285/06; Switzerland).

\section{Study procedure}

Figure 1 depicts the study design. First, a medical examination ensured that all infants were physically and physiologically healthy (healthy controls; infants with OFC) and that there were no health issues such as fever, infections, or similar to discourage surgical intervention (infants with OFC).

Next, infants' sleep was assessed for 3-4 consecutive days via actigraphs as outlined in more detail in the following paragraphs. In parallel, the first morning after sleep registration, saliva was sampled to assess cortisol values (see following paragraphs). Afterwards, infants with OFC underwent surgery. The period of surgery was 3-4 days with continuous sleep assessment, whereas saliva samplings were performed in the morning of the day of surgery. During this period, healthy controls had an assessment break of 3-4 days. The third/fourth day after surgery, sleep assessment continued for both groups for a further 3-4 days. Saliva sampling was performed in the OFC group on the morning of the third day postsurgery. On the last day of sleep assessment, saliva sampling was again performed in both the OFC and the control group.

\section{Assessment of infants' sleep and cortisol secretion}

\section{Sleep assessment}

Following a procedure described previously, ${ }^{20,21}$ sleep was assessed in two ways. First, mothers were trained to keep a daily log of their infant's sleep. They indicated for each 30-minute period of a day whether the infant was sleeping. Sleep was defined as follows: the child's eyes are closed; breathing is regular and rhythmical; and body, head, and limbs move only incidentally. Mothers additionally noted whether any change occurred in sleep routine. The information derived from the daily sleep log was also used to check possible discrepancies with, or missing data from, the actigraphy. ${ }^{19-21,23}$ The sleep log was also kept during the entire study period.

Second, sleep was objectively assessed under at-home conditions for 3-4 consecutive days and nights. According to Sadeh et $\mathrm{al}^{24}$ at-home sleep assessment in children has the advantage that sleep does not seem to be negatively affected. Infants wore a digital movement-measuring instrument (actigraph; SOMNOwatch ${ }^{\circledR}$; SOMNOmedics, Randersacker, Germany) on the left ankle. This commercially available tool has the dimension of a wristwatch and registers every movement above $0.012 \mathrm{~g}$ in a biaxial direction. The data, recorded in 30-second intervals, were digitally integrated and translated into sleep measures using the software program (based on sleep/wake algorithm as defined by Gorny et $\mathrm{a}^{25}$ ) provided by SOMNOwatch ${ }^{\circledR}$. Mothers were fully instructed in use of the device. They were trained to push the "marker button" to indicate the beginning and the end of every sleeping period;

\begin{tabular}{|c|c|c|c|c|c|c|c|c|c|c|c|c|}
\hline & & $\begin{array}{l}5 \text { days } \\
\text { before } \\
\text { surgery }\end{array}$ & $\begin{array}{l}4 \text { days } \\
\text { before } \\
\text { surgery }\end{array}$ & $\begin{array}{l}3 \text { days } \\
\text { before } \\
\text { surgery }\end{array}$ & $\begin{array}{l}2 \text { days } \\
\text { before } \\
\text { surgery }\end{array}$ & $\begin{array}{l}1 \text { day } \\
\text { before } \\
\text { surgery }\end{array}$ & $\begin{array}{l}\text { Day of } \\
\text { surgery }\end{array}$ & $\begin{array}{l}1 \text { day } \\
\text { after } \\
\text { surgery }\end{array}$ & $\begin{array}{l}2 \text { days } \\
\text { after } \\
\text { surgery }\end{array}$ & $\begin{array}{l}3 \text { days } \\
\text { after } \\
\text { surgery }\end{array}$ & $\begin{array}{l}4 \text { days } \\
\text { after } \\
\text { surgery }\end{array}$ & $\begin{array}{l}5 \text { days } \\
\text { after } \\
\text { surgery }\end{array}$ \\
\hline Variable & Group & & & & & & & & & & & \\
\hline \multirow{2}{*}{$\begin{array}{l}\text { Morning } \\
\text { cortisol }\end{array}$} & OFC & $x$ & & & & & $x$ & & & $\mathrm{X}$ & & $x$ \\
\hline & Controls & $x$ & & & & & & & & & & $x$ \\
\hline $\begin{array}{l}\text { Sleep } \\
\text { actigraphy }\end{array}$ & Controls & \multicolumn{4}{|c|}{ Aggregated data of $3-4$ days } & & & & \multicolumn{4}{|c|}{ Aggregated data of $3-4$ days } \\
\hline & & & & & & & \multicolumn{2}{|c|}{ Day of surgery } & & & & \\
\hline
\end{tabular}

Figure I Study overview.

Note: $X=$ assessment.

Abbreviation: OFC, orofacial cleft. 
that is to say, they pushed the "marker button" whenever the infant fell asleep and whenever the infant woke up. This instruction was important because an actigraph computes sleep/wake patterns as a function of the wearer's bodily activity. The actigraph identifies sleep when after a specific algorithm no further activity is registered. For example, the actigraph can erroneously identify the state of a calm but awake infant as sleep. Correspondingly, the actigraph can register activity and identify the state of a sleeping infant as awake when the infant is, in fact, sleeping in a baby carriage or in a baby seat while being driven in a car. To avoid false hits, mothers were carefully trained to use the actigraph. If unsure whether they had pushed the button firmly enough, mothers were instructed to push the button a second time. As in previous studies, ${ }^{20,21}$ to become accustomed to the actigraph, infants started wearing the instrument during an afternoon. Actigraphic measurements have been repeatedly shown to provide reliable data ${ }^{23,24}$ comparable with electroencephalography data of infants aged $<6$ months. ${ }^{26}$

The following sleep continuity parameters were collected: total sleep time during the day and at night and number of awakenings during the day and at night. Interclass correlation of night-to-night and day-to-day reliability was very high, $r>0.90$. Thus, data for the first 3 days and nights (first time point) and for the second 3 days and nights (second time point) were each combined to form composite variables.

\section{Assessment of the HPA axis system}

Morning cortisol levels have been shown to be a reliable biological marker for the HPA activity of infants, ${ }^{20,21,27}$ adolescents, and adults when measured repeatedly with strict reference to the time of awakening. ${ }^{28,29}$ Therefore, four saliva cortisol samples were collected by the mother after the last night of actigraphy in the morning at $0,10,20$, and 30 minutes after the infant's awakening and stored in a refrigerator. Waking times ranged from 8 am to 9 am. Feeding occurred only after saliva collection. When it turned out that saliva sampling on a given morning was too difficult (infant was too hungry, irritable; other issues), saliva sampling was repeated the following morning. The mothers were instructed and trained in saliva sampling before starting the study. Specifically, the mothers were trained to keep their infants in their lap in an upright position. Cotton swabs were provided with a tear-resistant nylon yarn. The mother wrapped the end of this nylon yarn around one of her fingers and the cotton swab was placed in the infant's mouth for about 1 minute. This procedure ensured that the cotton swab could not be swallowed in error.

\section{Saliva cortisol sampling technique and cortisol analysis}

Saliva samples were obtained as described. These were then returned to the laboratory, where samples were centrifuged at $4{ }^{\circ} \mathrm{C}(2,000 \mathrm{rpm}, 10$ minutes $)$ and stored at $-20^{\circ} \mathrm{C}$ until assay.

Free salivary cortisol concentrations were analyzed using a time-resolved immunoassay with fluorometric detection "Coat-A-Count" Cortisol RIA from Diagnostics Products Corporation (obtained through H. Biermann GmbH, Bad Nauheim, Germany) as described in detail elsewhere. ${ }^{30}$ Intraand interassay variabilities of this assay were less than $3.5 \%$ and $5.1 \%$, respectively.

The mean (and SD) was calculated for the cortisol values sampled at $0,10,20$, and 30 minutes after awakening. ${ }^{27}$

\section{Statistical analysis}

To compare both cortisol values and sleep parameters between the two groups over time (two time points), a series of analyses of variance (ANOVAs) for repeated measures was performed. Next, and more specifically, to calculate changes in cortisol values among the infants with OFC and undergoing OFC surgery, a further ANOVA for repeated measures was performed. The same statistical procedure was also applied to calculate changes in sleep patterns within the OFC surgery group. Post hoc analyses were performed using Bonferroni-Holm corrections for $P$-values. In case of deviations of sphericity, ANOVAs were performed using Greenhouse-Geisser corrected degrees of freedom $(d f)$, though the original $d f$ were reported with the relevant Greenhouse-Geisser epsilon value $(\varepsilon)$. For ANOVAs, effect sizes are indicated with the partial eta squared $\left(\eta^{2}\right)$, with $0.059 \geq \eta^{2} \geq 0.01$ indicating small (S), $0.139 \geq \eta^{2} \geq 0.06$ indicating medium (M), and $\eta^{2} \geq 0.14$ indicating large (L) effect sizes.

A series of correlations was performed between cortisol values and sleep variables.

The nominal $\alpha$ level was set at 0.05 . Statistical analyses were performed with SPSS $^{\circledR} 20.0$ (IBM Corporation, Armonk, NY, USA) for Apple MacIntosh ${ }^{\circledR}$.

\section{Results \\ Cortisol values over time and between infants with OFC and healthy controls}

Table 1 gives the descriptive and statistical data for cortisol values over time and separately for infants with OFC and healthy controls. 


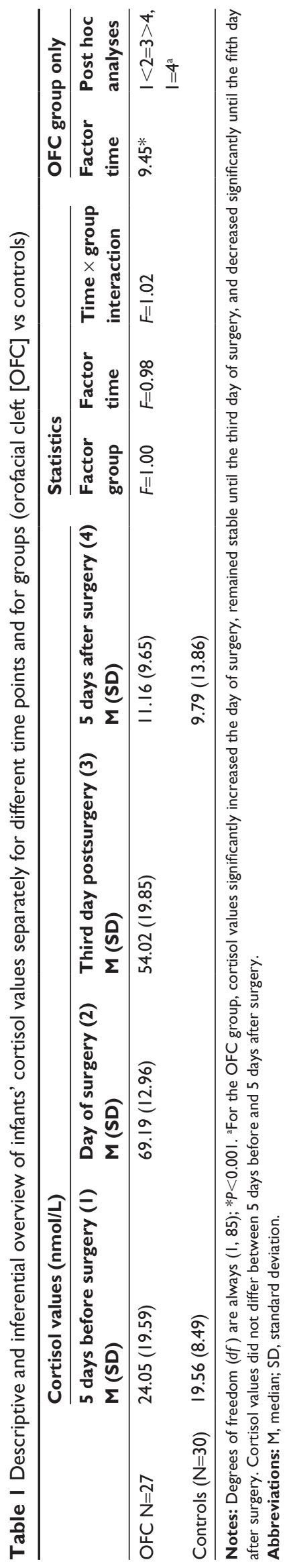

Cortisol values did not change between presurgery and postsurgery periods, and cortisol values did not differ between infants with OFC and healthy controls.

Cortisol values over time in infants with OFC and undergoing OFC surgery Cortisol values dramatically increased on the day of surgery and remained elevated until the third day after surgery; cortisol values reverted to baseline values 5 days after surgery (see Table 1).

\section{Sleep variables over time and between infants with OFC and healthy controls}

Table 2 gives the descriptive and statistical data for sleep values over time and separately for infants with OFC and healthy controls.

Sleep values did not change over time and did not differ between infants with OFC and healthy controls.

\section{Sleep variables over time in infants with OFC and undergoing OFC surgery}

Sleep variables dramatically changed on the day of surgery and remained unstable until the second day after surgery (data not shown), though these variables reverted to baseline values after the third day and remained stable until the end of the assessment (fifth day; see Table 2).

Correlations between cortisol values and sleep values in infants with OFC and healthy controls

Cortisol values and sleep parameters correlated moderately in healthy controls and in infants with OFC during the periods before and after surgery ( $r=-0.29$ to $-0.36, P<0.02)$.

\section{Correlations between cortisol values and sleep values in infants with OFC during the period of surgery}

Cortisol values and sleep parameters correlated significantly in infants with OFC during the surgery period; higher cortisol values were associated with poorer sleep variables $(r=-0.32$ to $-0.40, P<0.01)$.

\section{Discussion}

The key findings of the present study were that cortisol values and sleep patterns of infants with OFC and undergoing OFC surgery did not differ from cortisol values and sleep patterns of healthy controls. Additionally, in infants with OFC and 


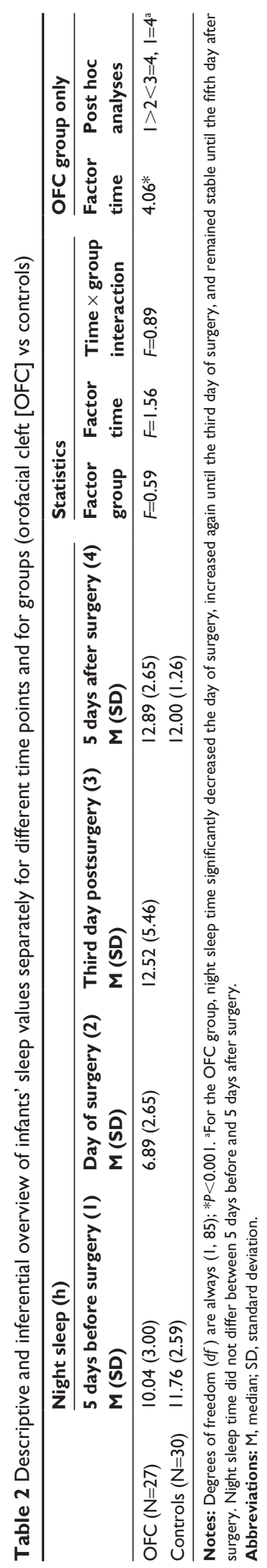

undergoing OFC surgery, both cortisol values and sleep patterns dramatically altered over the period of the surgery but returned to baseline values 5 days after surgical intervention. Therefore, data indicate that the cleft malformation does not expose the infant to an increased stress level. This finding reassures parents and is helpful for a normal infant-parent bonding - despite the possibly intimidating facial appearance. Additionally, data show no evidence that a single surgical intervention for OFC at the age of 5 months might produce any long-term damage in sleep and physiological processes such as the HPA axis. Accordingly, it seems highly unlikely that OFC surgery may be considered a traumatic life event during early infancy. ${ }^{22,31,32}$

Three hypotheses were formulated and each of these is considered in turn. First, we expected increased cortisol secretions in infants with OFC immediately during peri- and postsurgery, but not 5 days before or 5 days after surgery, and these expectations were supported. Therefore, we were able to confirm previous findings, ${ }^{11,12}$ though we were also able to expand on these in that the pattern of results was observed among 5-month-old infants undergoing surgery for OFC. Accordingly, our data lend further support to the notion that physiological stressors such as a surgical intervention do dramatically alter HPA axis activity in infants but that the elasticity of the HPA axis is such that 5 days poststimulation (here: surgery), cortisol secretion levels return to baseline.

Our second hypothesis was that the pattern of cortisol secretion of infants with OFC would not differ from that of healthy counterparts, and this was fully supported. Therefore, in our opinion, this pattern of results further shows the elasticity of the HPA axis and the observation that infants with OFC and undergoing surgery are not at increased risk for continuing deterioration of the HPA axis. Further, OFC surgery does not seem to be a stressor with long-term consequences. In contrast, Essex et $\mathrm{al}^{32}$ reported that other types of early life stress such as maternal depression, paternal depression, and family expressed anger may have long-term deleterious consequences in later childhood and in adolescence. Likewise, Halligan et $\mathrm{al}^{31}$ reported that infants exposed to mothers' postnatal depression were at increased risk of showing deteriorated HPA axis activity (ie, increased cortisol secretion) during adolescence. Further, Van den Bergh et al $^{33}$ reported that exposure to antenatal maternal anxiety led to deteriorated HPA axis activity and depressive symptoms in adolescence. Again, taking into account these other findings, our view is that OFC surgery on 5-month-old infants does not seem to cause adverse effects in the short term, and most probably also not in the longer term. In these regards, 
Gassling et $\mathrm{al}^{34}$ showed that, against expectations, adults with repaired OFC had significantly better stress-coping strategies and a faster cortisol return to baseline conditions than their healthy peers.

Our third hypothesis was that objectively assessed sleep and cortisol secretion would be associated, though the pattern of results did not fully support this hypothesis, in that a more fine-grained analysis was necessary to describe and interpret the pattern of results. On the one hand, and more specifically, cortisol secretion and sleep before and after surgery, between subsamples and across the sample as a whole, were only marginally associated. Accordingly, the present data are at odds with evidence showing poor sleep and increased cortisol secretion to be associated in 8-week-old infants. ${ }^{21}$ On the other hand, however, and more specifically, this pattern of poor sleep and increased cortisol secretion was observed specifically in infants with OFC during the perisurgical period. Poor sleep and increased cortisol secretion did correlate. From the overall pattern of results, we conclude that under most conditions, sleep and cortisol secretion are only weakly associated, probably because of low overall stimulation, while under conditions such as surgery (the present study) or intense somatic pain (infants suffering from infantile colic; Brand et $\mathrm{al}^{21}$ ), the association between poor sleep and deteriorated cortisol secretion is more evident.

Despite the intriguing results, several limitations warrant against overgeneralization. Firstly, the samples are rather small; thus, from a statistical point of view, the calculations might be underpowered. Secondly, all the infants with OFC and the healthy controls were recruited from the same university hospital and the same geographical and socioeconomic area; accordingly, a systematic sample bias cannot be excluded. Thirdly, the pattern of results might have emerged due to further but unassessed latent physiological and psychological variables. For example, infants' psychosocial development and parents' interactional behavior were not taken into account. Future studies might therefore introduce as additional possible confounders parents' strategies to cope with children with OFC, and parents' (and particularly mothers') psychological states. ${ }^{31-33}$ Fourthly, infants with OFC and undergoing OFC surgery were treated with anesthetic and analgesic, and the influence of these medications on sleep and cortisol secretion could not be statistically or experimentally controlled. Fifthly, saliva sampling during the period of surgery was not possible, as the oral cavity was contaminated with blood. Sixthly, data do not allow predictions as to infants' physiological and psychosocial development in the longer term. Seventhly, future studies might change the statistical approach in performing multilevel models testing lead-lag associations. Lastly, and most importantly, no conclusion can be made about "stress-related" increases over the course of the study, since no proper control condition was implemented. The authors only have comparison data from pre- and postsurgery time points but not from time points around the time of surgery. Whereas for obvious reasons no real comparable event in the healthy control group could be performed, future studies might apply a "natural stressor" (eg, regular medical examination) as a comparison condition. Accordingly, without any information on how a "normal" child would respond to a specific event, no conclusion can be drawn on the specificity of any potential responses to OFC surgery. However, it was most important to us to show that both sleep and cortisol secretion returned to baseline 5 days after OFC surgery and that, accordingly, adverse long-term effects of OFC surgery are unlikely.

\section{Conclusion}

Among a sample of infants with OFC and undergoing OFC surgery, cortisol secretion and sleep did not differ between 5 days before and 5 days after surgery, or from those of healthy controls. We conclude that OFC surgery undertaken at the age of 5 months does not seem to have long-term adverse physiological consequences.

\section{Acknowledgments}

We thank Nadine Sammer for data gathering and data entering. We also thank Nick Emler (University of Surrey, UK) for proofreading the manuscript. We further thank the Gottfried and Julia Bangerter-Ryhner Foundation for the unrestricted grant (SB) and the Austrian Cleft Palate Craniofacial Association for the Helene-Matras Award in the year 2008 (AAM).

\section{Disclosure}

The authors report no conflicts of interest in this work.

\section{References}

1. Mossey PA, Little J, Munger RG, Dixon MJ, Shaw WC. Cleft lip and palate. Lancet. 2009;374:1773-1785.

2. Eurocat-network.eu [homepage on the Internet]. European Surveillance of Congenital Anomalies (Eurocat). Available from: http://www.eurocatnetwork.eu. Accessed September 3, 2014.

3. Dixon MJ, Marazita ML, Beaty TH, Murray JC. Cleft lip and palate: understanding genetic and environmental influences. Nat Rev Genet. 2011; 12:167-178.

4. Brand S, Mueller AA. Quality of life among children, adolescents and adults with orofacial clefts. In: Stone JH, Blouin M, editors. International Encyclopedia of Rehabilitation. 2010. Available from: http://cirrie buffalo.edu/encyclopedia/en/article/294/. Accessed September 3, 2014. 
5. Sheldon SH. Sleep in infants and children. In Lee-Chiong TL, Sateia MJ, Carskadon MA, editors. Sleep Medicine. Philadelphia, PA: Hanley and Belfus, Inc.; 2004;99-103.

6. Davis KF, Parker KP, Montgomery GL. Sleep in infants and young children: Part one: Normal sleep. J Pediatric Health Care. 2004;18: 65-71.

7. Potts AL, Cheeseman JF, Warman GR. Circadian rhythms and their development in children: implications for pharmacokinetics and pharmacodynamics in anesthesia. Paediatric Anaesthesia. 2011;21: $238-246$.

8. Tollenaar MS, Jansen J, Beijers R, Riksen-Walraven JM, de Weerth C. Cortisol in the first year of life: normative values and intra-individual variability. Early Hum Dev. 2010;86:13-16.

9. Davis EP, Granger DA. Developmental differences in infant salivary alpha-amylase and cortisol responses to stress. Psychoneuroendocrinology. 2009;34:795-804.

10. Jansen J, Beijers R, Risken-Walraven M, de Weerth C. Cortisol reactivity in young infants. Psychoneuroendocrinology. 2010;35:329-338.

11. Gruber EM, Laussen PC, Casta A, et al. Stress response in infants undergoing cardiac surgery: a randomized study of fentanyl bolus, fentanyl infusion, and fentanyl-midazolam infusion. Anesth Analg. 2001;92:882-890.

12. Nakamura M, Suita S, Yamanouchi T, Masumoto K, Ogita K, Taguchi S, Uesugi T. Cortisol and cytokine responses after surgery in different age groups of pediatric patients. Pediatr Surg Int. 2003;19:194-199.

13. Gunnar MR, Talge NM, Herrera A. Stressor paradigms in developmental studies: what does and does not work to produce mean increases in salivary cortisol. Psychoneuroendocrinology. 2009;34:953-967.

14. Mantagos S, Moustogiannis A, Vagenakis AG. Diurnal variation of plasma cortisol levels in infancy. J Pediatr Endocrinol Metab. 1998;11: 549-553.

15. Gunnar MR, Brodersen L, Nachmias M, Buss K, Rigatuso J. Stress reactivity and attachment security. Dev Psychobiol. 1996;29:191-204.

16. Larson MC, White BP, Cochran A, Donzella B, Gunnar M. Dampening of the cortisol response to handling at 3 months in human infants and its relation to sleep, circadian cortisol activity, and behavioral distress. Dev Psychobiol. 1998;33:327-337.

17. Hatzinger M, Hemmeter UM, Brand S, Ising M, Holsboer-Trachsler E. Electroencephalographic sleep profiles in treatment course and longterm outcome of major depression: association with DEX/CRH-test response. J Psychiatr Res. 2004;38:453-465.

18. Buckley TM, Schatzberg AF. On the interactions of the hypothalamicpituitary-adrenal (HPA) axis and sleep: normal HPA axis activity and circadian rhythm, exemplary sleep disorders. J Clin Endocrinol Metab. 2005;90:3106-3114.

19. Hatzinger M, Brand S, Perren S, Stadelmann S, Wyl AV, Klitzing KV, Holsboer-Trachsler E. Sleep actigraphy pattern and behavioral/ emotional difficulties in kindergarten children: association with hypothalamic-pituitary-adrenocortical (HPA) activity. J Psychiatr Res. 2010;44:253-261.

20. Brand S, Furlano R, Sidler M, Schulz J, Holsboer-Trachsler E. 'Oh, baby, please don't cry!': in infants suffering from infantile colic hypothalamic-pituitary-adrenocortical axis activity is related to poor sleep and increased crying intensity. Neuropsychobiology. 2001;64:15-23.
21. Brand S, Furlano R, Sidler M, Schulz J, Holsboer-Trachsler E. Associations between infants' crying, sleep and cortisol secretion and mother's sleep and well-being. Neuropsychobiology. 2014;69:39-51.

22. Shea A, Walsh C, Macmillan H, Steiner M. Child maltreatment and HPA axis dysregulation: relationship to major depressive disorder and post traumatic stress disorder in females. Psychoneuroendocrinology. 2005;30:162-178

23. El-Sheikh M, Buckhalt JA, Mark Cummings E, Keller P. Sleep disruptions and emotional insecurity are pathways of risk for children. JChild Psychol Psychiatry. 2007;48:88-96.

24. Sadeh A, Lavie P, Scher A, Tirosh E, Epstein R. Actigraphic homemonitoring sleep-disturbed and control infants and young children: a new method for pediatric assessment of sleep-wake patterns. Pediatrics. 1991;87:494-799.

25. Gorny SW, Allen RP, Krausmann DT, Cammarata J, Earley CJ. A parametric and sleep hysteresis approach to assessing sleep and wake from wrist activity meter with enhanced frequency range. Paper presented at the eleventh annual meeting of the Associated Professional Sleep Societies; June 10-15, 1997; San Francisco, CA, USA.

26. So K, Buckley P, Adamson TM, Horne RS. Actigraphy correctly predicts sleep behavior in infants who are younger than six months, when compared with polysomnography. Pediat Res. 2005;58:761-765.

27. Tegethoff M, Knierzinger N, Meyer AH, Meinlschmidt G. Cortisol awakening response in infants during the first six postnatal months and its relation to birth outcome. Psychoneuroendocrinology. 2013;38: 629-637.

28. Hellhammer DH, Wüst S, Kudielka BM. Salivary cortisol as a biomarker in stress research. Psychoneuroendocrinology. 2009;34:163-171.

29. Pruessner JC, Wolf OT, Hellhammer H, et al. Free cortisol levels after awakening: a reliable biological marker for the assessment of adrenocortical activity. Life Sci. 1997;61:2539-2549.

30. Dressendörfer RA, Kirschbaum C, Rohde W, Stahl F, Strasburger CJ. Synthesis of a cortisol-biotin conjugate and evaluation as a tracer in an immunoassay for salivary cortisol measurement. J Steroid Biochem Mol Biol. 1992;43:683-692.

31. Halligan SL, Herbert J, Goodyer IM, Murray L. Exposure to postnatal depression predicts elevated cortisol in adolescent offspring. Biol Psychiatry. 2004;55:376-381

32. Essex MJ, Shirtcliff EA, Burk LR, et al. Influence of early life stress on later hypothalamic-pituitary-adrenal axis functioning and its covariation with mental health symptoms: a study of the allostatic process from childhood into adolescence. Dev Psychopathol. 2011;23:1039-1058.

33. Van den Bergh BR, Van Calster B, Smits T, Van Huffel S, Lagae L. Antenatal maternal anxiety is related to HPA-axis dysregulation and self-reported depressive symptoms in adolescence: a prospective study on the fetal origins of depressed mood. Neuropsychopharmacology. 2008;33:536-545.

34. Gassling V, Holterhus PM, Herbers D, et al. Stress-coping and cortisol analysis in patients with non-syndromic cleft lip and palate: an explorative study. PLoS One. 2012;7:e41015.
Neuropsychiatric Disease and Treatment

\section{Publish your work in this journal}

Neuropsychiatric Disease and Treatment is an international, peerreviewed journal of clinical therapeutics and pharmacology focusing on concise rapid reporting of clinical or pre-clinical studies on a range of neuropsychiatric and neurological disorders. This journal is indexed on PubMed Central, the 'PsycINFO' database and CAS,
Dovepress

and is the official journal of The International Neuropsychiatric Association (INA). The manuscript management system is completely online and includes a very quick and fair peer-review system, which is all easy to use. Visit http://www.dovepress.com/testimonials.php to read real quotes from published authors. 